\title{
Effect of superimposed compressive stresses on rolling contact fatigue initiation at hard and soft inclusions
}

\author{
Mahdavi, Hamidreza; Poulios, Konstantinos; Niordson, Christian F.
}

Published in:

International Journal of Fatigue

Link to article, DOI:

10.1016/j.ijfatigue.2019.105399

Publication date:

2020

Document Version

Peer reviewed version

Link back to DTU Orbit

Citation (APA):

Mahdavi, H., Poulios, K., \& Niordson, C. F. (2020). Effect of superimposed compressive stresses on rolling contact fatigue initiation at hard and soft inclusions. International Journal of Fatigue, 134, [105399].

https://doi.org/10.1016/j.ijfatigue.2019.105399

\section{General rights}

Copyright and moral rights for the publications made accessible in the public portal are retained by the authors and/or other copyright owners and it is a condition of accessing publications that users recognise and abide by the legal requirements associated with these rights.

- Users may download and print one copy of any publication from the public portal for the purpose of private study or research.

- You may not further distribute the material or use it for any profit-making activity or commercial gain

- You may freely distribute the URL identifying the publication in the public portal 


\title{
Effect of superimposed compressive stresses on rolling contact fatigue initiation at hard and soft inclusions
}

\author{
Hamidreza Mahdavi*, Konstantinos Poulios, Christian F. Niordson \\ Department of Mechanical Engineering, Section of Solid Mechanics, Technical University \\ of Denmark, Nils Koppels Allé, Building 404, DK-2800 Kgs. Lyngby
}

\begin{abstract}
A semi-analytical framework is introduced for the evaluation of favorable residual stresses for delaying rolling contact fatigue initiation. Subsurface stress histories are applied to a micro-scale model accounting for isolated inclusions of different types and geometries. Micro-scale von Mises stresses are calculated based on Eshelby's method and considered as an estimator of crack initiation due to plastic deformation. The most critical cases in terms of micro-scale plasticity are identified and the effect of macro-scale compressive residual stresses is considered. Finally, optimized residual stresses are determined that minimize the maximum attained micro-scale von Mises stress at different depths.
\end{abstract}

Keywords: Rolling contact fatigue, Non-metallic inclusions, Residual stresses, Fatigue damage initiation, Eshelby's method

\section{Introduction}

Rolling element bearings, widely used to support rotary elements in modern machinery, mainly suffer from two failure mechanisms corresponding to surface originated pitting and subsurface initiated spalling [1,2]. While surface initiated pitting is normally attributed to poor lubrication conditions [3], well-lubricated bearings with smooth contact surfaces typically fail due to subsurface initiated rolling contact fatigue with cracks emanating from non-metallic inclusions $[1,4]$. With continuously emerging applications in

\footnotetext{
${ }^{*}$ Corresponding author: hamah@mek.dtu.dk
} 
new types of machinery, new environments and evolving bearing steel technologies, research for accurately predicting and extending bearing lifetime is highly important.

Contributions to a better understanding, quantification and improvement of rolling contact fatigue include i) experimental work for determining the fatigue performance of bearing steels [5,6], ii) lifetime models at the component level $[7,8]$, iii) phenomenological fatigue models at the homogenized material level $[9,10]$, and iv) micro-mechanical models for damage evolution typically in the vicinity of inclusions [11, 12].

Despite the big progress in understanding the fatigue failure of bearings, there are still often contradicting theories and interpretations of experimental data. This partial lack of consensus [7] indicates that a full understanding of the links between all phenomena observed in the aforestated research areas still has to be reached. The main difficulties for reaching a full understanding of rolling contact fatigue include:

- complex spatial and temporal variations of the subsurface stress state,

- varying material microstructure with material depth [13] and time [14],

- stochastic occurrence of inclusions of different types, and

- subsurface stress histories offset by an unknown reference stress state.

Especially with regard to the latter point, depth dependent residual and hoop stresses of significant magnitude compared to the material strength are not uncommon [13]. Residual stresses typically arise from phase transformations during heat treatment or operation but they can also be induced or relaxed by plastic deformation during manufacturing or running-in of a bearing $[15,16]$. While any reported monotonic positive or negative effect of residual stresses on fatigue performance can only be valid within a certain range of values $[17,18,19]$, there are also theoretical models capturing a non-monotonic and hence more realistic dependence [9, 20, 21, 22, 16].

An often disputed aspect in rolling contact fatigue is the existence of a fatigue endurance limit and its relevance for lifetime calculations [7]. Nevertheless, based on the proposals by Ioannides and Harris [23, 24], the concept of a fatigue stress limit, defined as a stress level below which no material deterioration is expected, is now part of widely used bearing life models [8]. Assuming the existence of such a limit, micro-mechanical models have been developed to determine how this limit would be affected by inclusions, based for example on Eshelby's method [21, 25] or finite element analysis [26]. In 
any case, irrespective of the existence of a material fatigue limit, a better understanding of the early phase of crack development is certainly relevant for improving bearing life models.

Regarding fatigue crack initiation, cyclic plasticity both at the macroand micro-scale is often seen as a contributing factor to damage accumulation [14]. While cyclic plasticity observed at the macro-scale is mainly related to transformation of the softer retained austenite to martensite, it is stress concentrations near material inhomogeneities that lead to localized micro-scale plastic deformations. The latter case is concerned here, with the present work dealing with micro-scale plasticity in the vicinity of non-metallic inclusions. Although the density and size of inclusions in bearing steels can be reduced to a very large extent by appropriate steelmaking practices [27, 28], their occurance cannot be excluded entirely. Even for quite clean steels, inclusions of different forms, sizes and orientations occur stochastically leading stress concentration sites at the micro-scale. In that sense, a practical approach is to simply consider the worst case of inclusion type and shape for a given steel.

For a properly loaded bearing, it is in general expected that even if the yield limit of the material is initially exceeded locally near an inhomogeneity, the amount of near inclusion plasticity per load cycle will eventually decay to zero. A numerical simulation of such an elastic shakedown process after the initial exceeding of the material yield limit is rather complex and computationally expensive. However, predicting a conservative load range for which the material yield limit is not exceeded in the first place is rather simple. This fact has been exploited in [29] in order to predict a load limit, claimed to represent a fatigue endurance limit, which ensures that micro-scale plasticity does not occur in any phase of the bearing operation, not even initially.

Moreover, since the onset of plasticity is governed by the maximum von Mises stress attained during a complete overrolling, it is expected that any superimposed stresses at the component level, such as residual or hoop stresses, will affect the estimated fatigue endurance limit. This effect has clearly been demonstrated in [29] and is further pursued in the present work in order to determine optimal residual stresses as a function of the depth for different inclusion types and shapes. A simple semi-analytical method is proposed for determining a macro-scale residual stress that minimizes the maximum micro-scale von Mises at any time instant and any point around a subsurface inclusion of a given type and shape.

As input for the method, macroscopic subsurface stress histories at dif- 
ferent depths below a Hertzian line contact are extracted from a plane-strain finite element model. These are subsequently applied as far-field stresses for a 3D micro-scale model accounting for isolated inclusions of different geometries and orientations. Micro-scale stress histories around each considered inclusion are calculated efficiently by Eshelby's method. Finally, adopting the micro-scale von Mises stress as the optimization criterion for delaying microscale plasticity and thereby also damage accumulation, optimal compressive residual stresses added to the axial and circumferential stress components are determined as a function of depth for different types of inclusions.

\section{Analytical and numerical methods}

In the first part of this section, the analytical method employed to determine the micro-scale stress field, is explained. Then, a numerical procedure is presented, for obtaining optimal initial residual stresses that delay the onset of micro-scale plasticity as a function of depth from the surface.

\subsection{Micro-mechanical model}

In a typical roller bearing, subsurface stress histories at different depths $y$ into the bearing raceway can easily be computed for any given nominal Hertzian pressure $P_{H}$ in the roller-raceway contact. Assuming for instance, a frictionless line contact, a plane-strain finite element model with a theoretical parabolic Hertzian contact load can be used for extracting all relevant stress profiles at different depths as illustrated in Figure 1.

Such macroscopic stresses, obtained based on the assumption of an elastic and homogeneous material, can then be applied as far-field stresses to a micro-scale model accounting for the presence of non-metallic inclusions. This is a valid approach as long as the size of the assumed micro-scale inclusions is small enough compared to stress gradients at the macro-scale and the distance to the surface. In the present work, the four stress components included in Figure 1 are used for defining a 3D stress state transferred from the $2 \mathrm{D}$ plane-strain macro-scale model to the $3 \mathrm{D}$ micro-scale model. In both models, the coordinates $x, y$, and $z$ are respectively parallel to the circumferential, radial and axial directions of a bearing raceway. For a periodic load, a material point at a certain depth below the surface will repeatedly experience the four extracted macroscopic stress histories during every loading cycle.

Assuming a fully bonded elastic ellipsoidal inclusion inside an infinite elastic matrix at the micro-scale, Eshelby's method [30, 31] is extremely 


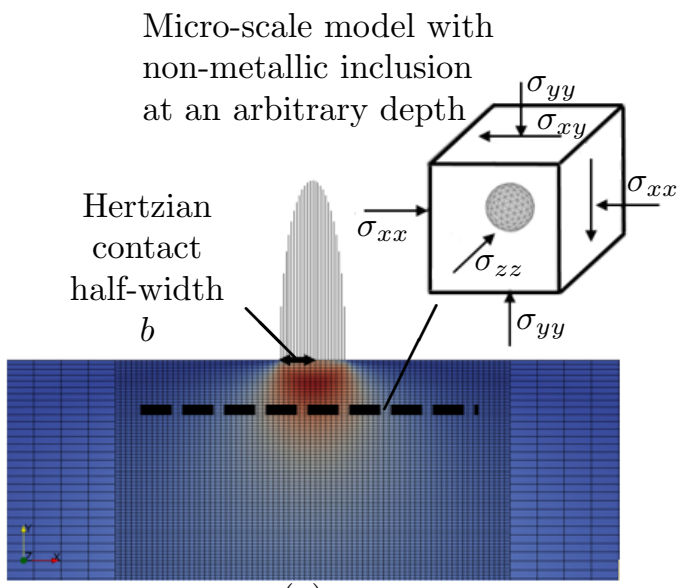

(a)

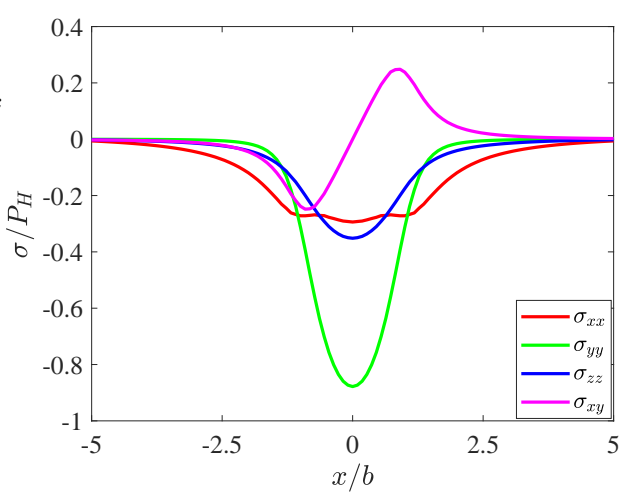

(b)

Figure 1: Determination of macroscopic stress histories: (a) Schematic view of the 2D plane strain finite element model, (b) Sub-surface stresses at depth $y=-0.54 b$.

efficient, compared to numerical methods, in providing the local stress field around the inclusion [21]. In particular, the implementation of Eshelby's method by Meng et al. [32] is the one used in this study to relate the farfield macroscopic stresses to local stresses around non-metallic inclusions.

Let $\sigma_{k l}(y, t)$ be the far-field stress tensor depending on the depth $y$ and time $t$, extracted from the aforementioned macro-scale plane-strain finite element model. Then, assuming an ellipsoidal inclusion at a specific depth $y$, the idea is to use Eshelby's method to find the corresponding stress field $\tilde{\sigma}_{i j}(P, y, t)$ covering all points $P$ around the inclusion at the micro-scale. Under the linearity assumption of Eshelby's theory, the far-field stress state $\sigma_{k l}$ can be converted to the microscopic one $\tilde{\sigma}_{i j}$ at each specific point $P$ around the inclusion with the help of a stress concentration tensor $T_{i j k l}^{\langle P\rangle}$ defined such that

$$
\tilde{\sigma}_{i j}(P, y, t)=T_{i j k l}^{\langle P\rangle} \sigma_{k l}(y, t) .
$$

In this linear mapping, the fourth order tensor $T_{i j k l}^{\langle P\rangle}$ is independent of time $t$ and depth $y$, and it only depends on the shape of the inclusion and the considered location $P$ around it. For a given inclusion shape, it is therefore sufficient to employ Eshelby's method with only six unit load cases corresponding to each independent component of $\sigma_{k l}$, in order to evaluate all 36 independent components of the stress concentration tensor $T_{i j k l}^{\langle P\rangle}$ for any point $P$, based on Eq. (1). In the plane strain case considered here, only four 
out of the six macro-scale components are active and the number of relevant tensor components is actually reduced to 24 . The described procedure can be applied as a pre-processing step, in order to precompute and store a set of tensors $T_{i j k l}^{\langle P\rangle}$ for multiple points $P$ around the inclusion. Later, in studies possibly involving multiple load cases and depths $y$, the stress state at any of the pre-treated points $P$ can easily be recovered by direct use of Eq. (1) at a minimal computational cost.

\subsection{Determination of optimal residual stresses}

The basic idea behind the optimization presented below is to delay the onset of plasticity at all points $P$ in the vicinity of an inclusion of a given shape. Ideally, the definition of a fatigue stress limit criterion would only require to ensure elastic shakedown, but avoiding the plastic deformation completely can also be seen as a conservative approach. In a purely elastic model, it is of course not possible to differentiate between possible steady state conditions such as elastic or plastic shakedown and ratcheting. However, the linear elasticity based maximum von Mises stress throughout a complete loading period is a valid criterion for avoiding micro-scale plasticity entirely and at the same time a conservative constraint for excluding cyclic plasticity. Minimizing the maximum elastic von Mises stress experienced by any point around a given inclusion through time is therefore chosen here as the optimization objective.

As recognized for example in [20] and [29], the presence of residual stresses will affect the von Mises stress history at a material point at the macroscale. This dependence actually carries over through Eq. (1) to the von Mises stresses at the micro-scale [21]. In fact, even a change in the hydrostatic stress at the macro-scale can affect the micro-scale von Mises stresses because the transformation according to Eq. (1) does not in general preserve the hydrostatic or deviatoric nature of a given stress state.

First, the macro-scale stress history $\sigma_{k l}(y, t)$ must be modified according to the presence of macro-residual stresses. Although for point contact bearings, subsurface radial residual stresses can reportedly be significant [14], neglecting the radial residual stress component in the central part of line contacts is a reasonable simplification [33]. Moreover, for an initial residual stress state imposed through heat treatment, the circumferential and axial components will be of similar magnitude. Hence, one rather common scenario of superimposed residual stresses $\sigma_{\text {res }}(y)$ at the macro-scale can be represented by the following shift in stress space 


$$
\hat{\sigma}_{k l}\left(y, t, \sigma_{\text {res }}(y)\right)=\sigma_{k l}(y, t)+\left[\begin{array}{ccc}
\sigma_{\text {res }}(y) & 0 & 0 \\
0 & 0 & 0 \\
0 & 0 & \sigma_{\text {res }}(y)
\end{array}\right]
$$

with the offset macro-scale stress $\hat{\sigma}_{k l}$ replacing the original one $\sigma_{k l}$. Other patterns of initial stress states, such as hoop stress due to mounting and residual stresses due to plastic deformation may be accounted for through modifications of Eq. (2).

For the macro-scale, the von Mises stresses $\sigma_{\mathrm{VM}}(y, t)$ and $\hat{\sigma}_{\mathrm{VM}}\left(y, t, \sigma_{\text {res }}(y)\right)$ can be calculated either for the unmodified stress state $\sigma_{k l}$ or for the modified one $\hat{\sigma}_{k l}$ that includes the superimposed residual stresses, respectively. Figure 2 shows the effect of adding a residual stress of $\sigma_{\text {res }}=-0.17 P_{H}$ on the two affected stress components according to Eq. (2) and the von Mises stress at the macro-scale at $y=-0.54 b$. It is evident that the significant reduction in the maximum attained von Mises stress can be seen as a means for suppressing or delaying the onset of plasticity.
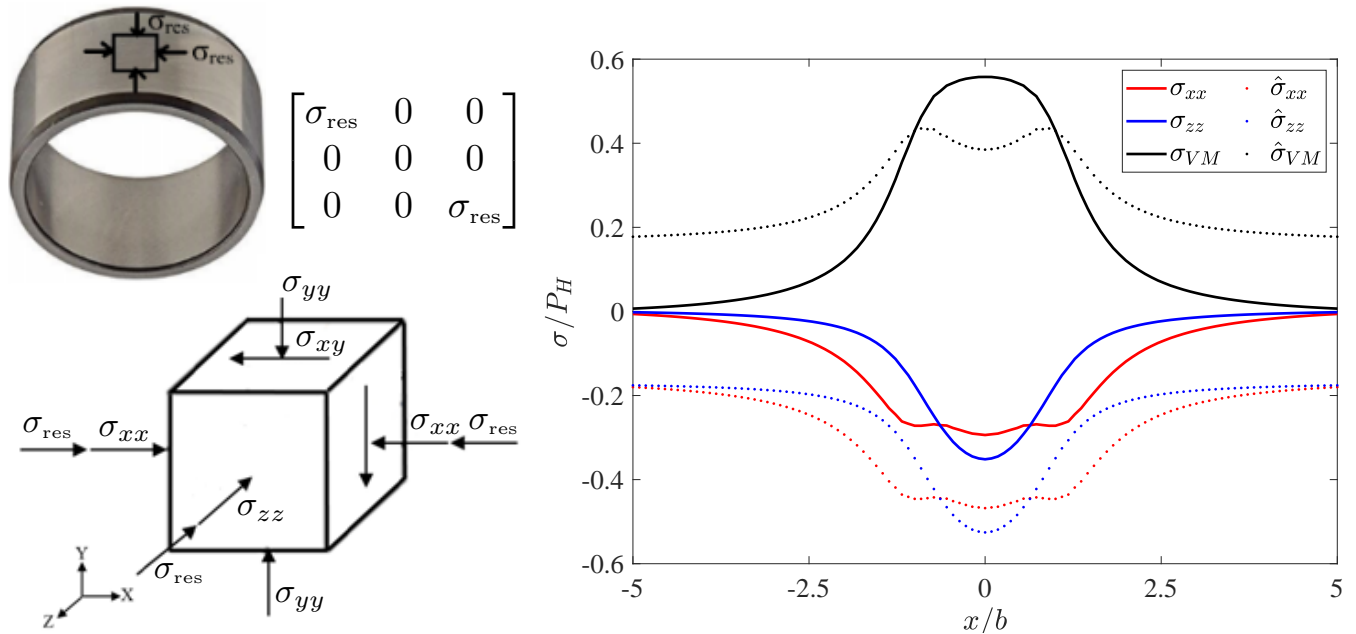

Figure 2: Effect of a residual stress $\sigma_{\text {res }}=-0.17 P_{H}$ on the macro-scale von Mises stresses at depth $y=-0.54 b$.

In practical applications, bearings will normally be dimensioned such that macro-scale stresses will anyway be safely below the material yield limit. Nevertheless, even if stresses at the macro-scale are well below the yield limit, stress concentrations around inclusions can still lead to plastic deformations 
locally. Therefore, the concept illustrated in Figure 2 for the macro-scale, becomes more relevant to apply at the micro-scale. In that manner, macroscale residual stresses could be exploited to avoid micro-scale plasticity close to an inclusion.

In order to account for the possibility of plasticity at the micro-scale, a practical approach is to consider a representative cloud of points around an inclusion as illustrated conceptually in Figure 3. Knowing the Eshelby based stress concentration tensors $T_{i j k l}^{\langle P\rangle}$ for any point $P$ in the cloud, the local stress tensor can be obtained as in Eq. (1), now including the effect of macro-scale residual stresses with

$$
\hat{\tilde{\sigma}}_{i j}=T_{i j k l}^{\langle P\rangle} \hat{\sigma}_{k l}
$$

which can finally lead to micro-scale von Mises stresses

$$
\sigma_{\mathrm{VM}}^{\langle P\rangle}\left(t, \sigma_{\mathrm{res}}\right)=\sqrt{\frac{\left(\hat{\tilde{\sigma}}_{11}-\hat{\tilde{\sigma}}_{22}\right)^{2}+\left(\hat{\tilde{\sigma}}_{22}-\hat{\tilde{\sigma}}_{33}\right)^{2}+\left(\hat{\tilde{\sigma}}_{33}-\hat{\tilde{\sigma}}_{11}\right)^{2}+6 \hat{\tilde{\sigma}}_{12}^{2}}{2}}
$$

where dependence on the depth $y$ is dropped for simplicity.

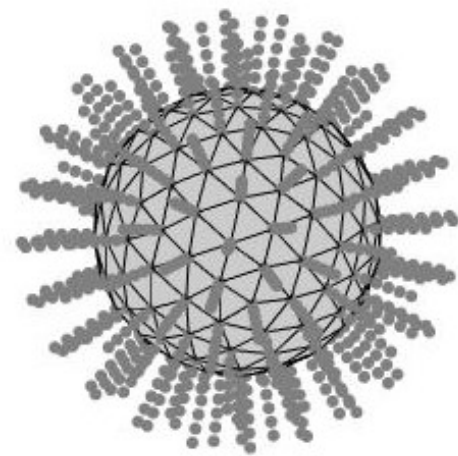

Figure 3: Representative cloud of points around a spherical inclusion.

The idea now is to determine an optimal value $\sigma_{\text {res }}^{*}$ of the compressive residual stress $\sigma_{\text {res }}$, at each depth $y$, that will minimize the maximum local von Mises stresses throughout a loading cycle and among all points around the inclusion. This optimization can be formulated as

$$
\sigma_{\text {res }}^{*} \leftarrow \min _{\sigma_{\text {res }}} \max _{t} \max _{P} \sigma_{\mathrm{vM}}^{\langle P\rangle}\left(t, \sigma_{\text {res }}\right) .
$$


This univariate minimization problem could be solved using any available single variable minimizer, but for simply demonstrating the concept, a brute force approach is also sufficient. In the present work, a range of values is simply swept for $\sigma_{\text {res }}$ with fixed increments. For any probed residual stress value, the time step $t$ and point $P$ around the inclusion are determined, which correspond to the maximum von Mises stress, according to the inner two max operators in Eq. (5). Then, the optimum $\sigma_{\text {res }}^{*}$ value is determined by comparing the obtained maximum von Mises stresses among all probed $\sigma_{\text {res }}$ values. It should be mentioned that the process has to be repeated at different depths $y$ from the surface, ultimately leading to an optimal residual stresses profile $\sigma_{\text {res }}^{*}(y)$.

Despite the simplicity of the proposed method, when it comes to applying it to real applications, there are a few practical aspects that require consideration. Two basic assumptions behind Eq. (2) is that the loading is defined through a unique Hertzian pressure $P_{H}$, and that macro-scale residual stresses remain constant. In reality, a material point in a bearing will experience a load spectrum where load peaks experienced occasionally may be considerable higher than the Hertzian pressure range that is representative for most of the lifetime. A deliberate choice of a specific load level to optimize for is necessary before applying the method. An alternative strategy could also be to optimize for different Hertzian pressures at different subsurface depths. Moreover, when implementing calculated optimal residual stresses in a bearing, it will possibly be necessary to compensate for effects like mounting stresses and phase transformations that may affect the actual residual stresses established after running in.

\section{Results and discussions}

In this section, bearing geometry and material parameters used in this study are provided, followed by maximum von Mises stress profiles calculated for different inclusions. Finally, optimal superimposed compressive residual stress profiles are reported.

\subsection{Geometry and material information}

An 81212 thrust bearing with the geometrical and mechanical properties given in Table 1 forms the basis for this study. At the micro-scale, two different types of inhomogeneities are investigated with aluminium oxide $\left(\mathrm{Al}_{2} \mathrm{O}_{3}\right)$ 
as a typical hard inclusion and manganese sulfide $(\mathrm{MnS})$ as a typical soft inclusion. For both types, the investigation performed considers inclusions of spherical form first, followed by inclusions of a prolate ellipsoidal form with an aspect ratio of 3 at different orientations, illustrated in Figure 4 . The considered inclusions have a typical size of 5-10 $\mathrm{km}[21,26,34]$. However, it is necessary to mention that the stress fields calculated by Eshelby's method are independent of the inclusion size, hence not accounting for size effects.

Table 1: Thrust bearing geometry and material parameters used in this study.

\begin{tabular}{cccccc}
\hline $\begin{array}{c}\text { Inner } \\
\text { diameter }\end{array}$ & $\begin{array}{c}\text { Outer } \\
\text { diameter }\end{array}$ & $\begin{array}{c}\text { Number } \\
\text { of rollers }\end{array}$ & $\begin{array}{c}\text { Roller } \\
\text { element } \\
\text { length }\end{array}$ & $\begin{array}{c}\text { Max. } \\
\text { Hertzian } \\
\text { pressure } \\
\left(P_{H}\right)\end{array}$ & $\begin{array}{c}\text { Hertzian } \\
\text { contact } \\
\text { half-width } \\
(b)\end{array}$ \\
\hline $60 \mathrm{~mm}$ & $95 \mathrm{~mm}$ & 20 & $10.5 \mathrm{~mm}$ & $2.3 \mathrm{GPa}$ & $0.22 \mathrm{~mm}$ \\
\hline
\end{tabular}

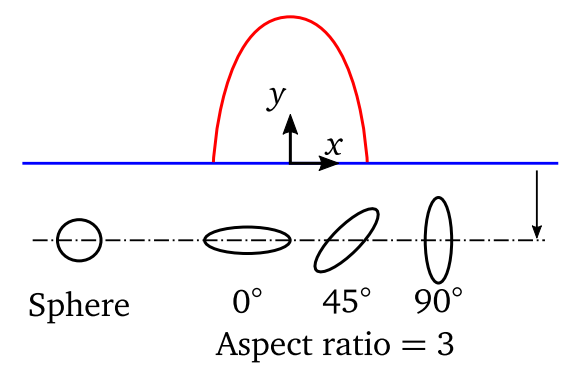

Figure 4: Schematic view of subsurface inclusions with respect to a Hertzian contact.

Regarding the material properties of the steel matrix, a Young's modulus of $203 \mathrm{GPa}$ and a Poisson's ratio of 0.3 are used while the corresponding values for aluminium oxide are $375 \mathrm{GPa}$ and 0.25 , respectively [26]. As manganese sulfide is known to yield at stresses as low as $90 \mathrm{MPa}$ at room temperature [35], plastic deformation can not simply be neglected for this inclusion type. Therefore, the lack of plasticity in the Eshelby based micro-mechanical model has to be compensated for, by determining effective elasticity parameters for this inclusion type based on a simple approximation of its elastoplastic response.

Under realistic loads and the actual elastic properties for manganese sulfide of $118 \mathrm{GPa}$ for Young's modulus and 0.23 for Poisson's ratio [26], a 
purely elastic solution predicts equivalent stresses in the inclusion well above the yield limit of $90 \mathrm{MPa}$. In reality, severe plastic deformation is expected where the inclusion will mainly contribute to its surrounding with its bulk modulus while shear stresses on the order of the yield limit will be very low compared to shear stresses in the surrounding steel matrix. Hence, a better elastic approximation of manganese sulfide inclusions under realistic loading could be reached by maintaining the original bulk modulus while reducing the shear modulus by at least one order of magnitude.

The actual elasticity parameters of manganese sulfide, mentioned above, lead to the following bulk and shear moduli

$$
K=\frac{E}{3(1-2 \nu)} \simeq 73 \mathrm{GPa} \text { and } \quad G=\frac{E}{2(1+\nu)} \simeq 48 \mathrm{GPa}
$$

Maintaining the same bulk modulus, but adopting a much lower effective shear modulus $G^{*}=0.0306 G$ leads to the effective elasticity parameters used for representing manganese sulfide inclusions in this study, evaluated as

$$
E^{*}=\frac{9 K G^{*}}{3 K+G^{*}} \simeq 4.37 \mathrm{GPa} \quad \text { and } \quad \nu^{*}=\frac{3 K-2 G^{*}}{2\left(3 K+G^{*}\right)} \simeq 0.49
$$

The validity of the approximation has been confirmed by comparing displacements and stress fields between an elastoplastic finite element simulation with the original elasticity parameters and a purely elastic simulation with the modified elasticity parameters.

\subsection{Von Mises stresses without superimposed compressive stresses}

The stress concentration effect of different inclusion types is investigated first without any additional macro-scale residual stresses. While a single maximum von Mises stress over time is representative for the onset of plasticity at each considered depth $y$ for the macro-scale, at the micro-scale, the entire field of von Mises stresses around a considered inclusion needs to be computed at each time step to determine a maximum over space and time. For practical purposes, micro-scale von Mises stresses are evaluated here at a large number of points around an inclusion in order to approximate the maximum. Repeating this procedure at 16 different depths from the surface up to approximately 2.5 times the contact half-width, the maximum von Mises stress profiles shown in Figures 5 and 6 are obtained for hard and soft inclusions, respectively. 


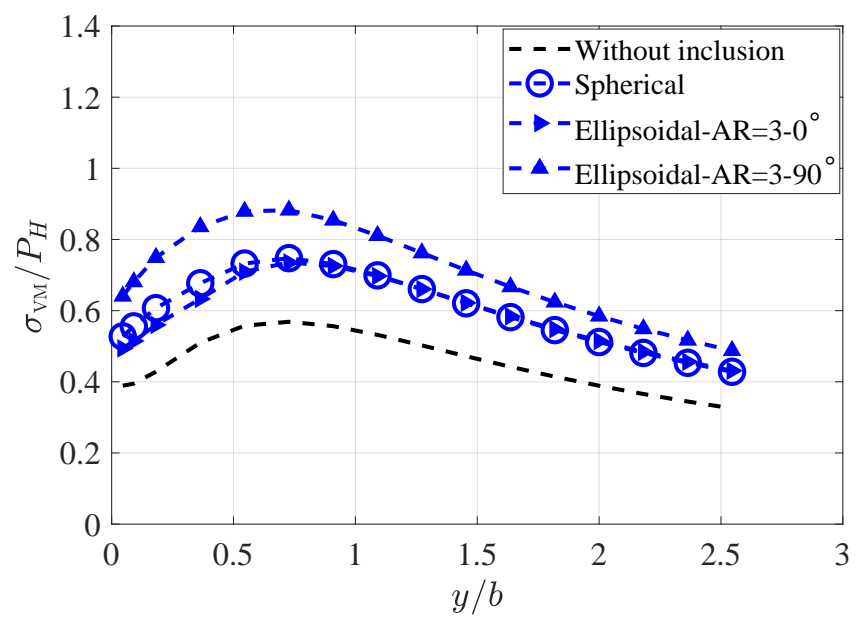

Figure 5: Von Mises stresses as a function of depth for the hard inclusions.

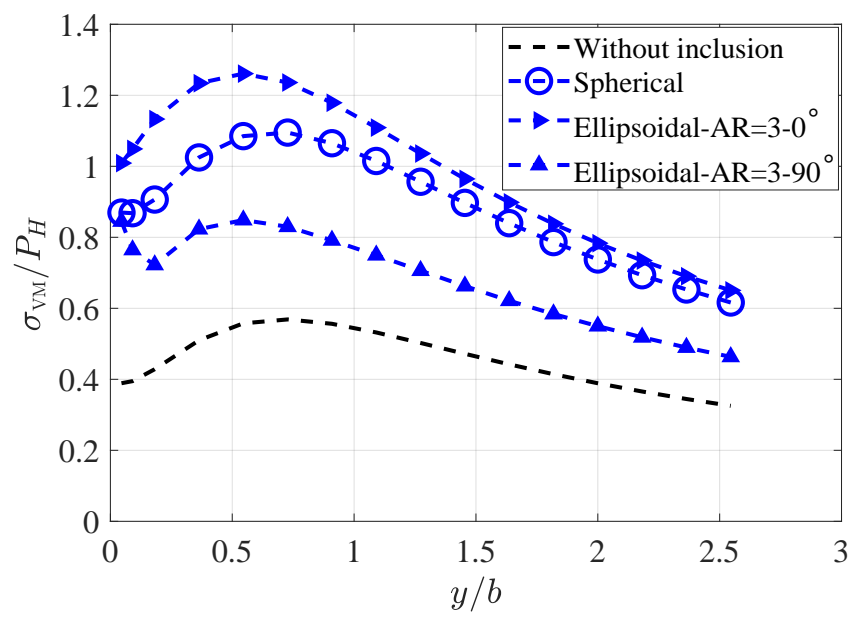

Figure 6: Von Mises stresses as a function of depth for the soft inclusions.

Apart from the spherical form, the graphs include two selected orientations of ellipsoidal inclusions, with the ellipsoid major axis either parallel to the rolling direction $\left(0^{\circ}\right)$ or perpendicular to the raceway surface $\left(90^{\circ}\right)$. According to the results of Figure 5 for hard inclusions, the case of inclusions elongated in the direction perpendicular to the surface leads to the highest von Mises value and is therefore more prone to micro-scale plasticity. On 
the contrary for soft inclusions, Figure 6 shows that ellipsoidal inclusions elongated along the rolling direction lead to higher von Mises stresses. It is seen that there is a considerably stronger stress concentration effect of soft inclusions on the maximum von Mises stresses when compared to hard inclusions. Hence, accounting for the very limited capacity of manganese sulfide to withstand shear loads due to its low yield limit, this type of inclusion appears to be more critical for micro-scale plasticity in the surrounding steel matrix. The often experimentally observed initiation of cracks around hard inclusions must therefore be attributed to debonding between inclusion and matrix. In particular, hard inclusions can either debond from the surrounding matrix during manufacturing, due to thermal expansion mismatch or severe deformation, or during the initial operation, due to the applied load. In both cases, the debonded hard inclusions lead to even stronger stress concentration governing damage initiation $[27,36]$. Nevertheless, results for fully bonded hard inclusions are still provided in the present work, similar to other studies from the literature [21], in order to put the presented effect of soft inclusions better into perspective.

Table 2 summarizes all maximum von Mises stresses relative to the applied Hertzian pressure, obtained for the aforementioned inclusion types and forms and additionally for different orientations between $0^{\circ}$ and $90^{\circ}$. The table also provides the relative increase in the attained von Mises stresses compared to the maximum value at the macro-scale, i.e. without the effect of any inclusion. Compared to the macro-scale maximum von Mises stress, the presence of a hard spherical inclusion leads to a maximum micro-scale von Mises stress increased by a factor of 1.31. For a hard ellipsoidal inclusion of aspect ratio equal to 3 , the corresponding stress increase factor varies from 1.29 to 1.55 for the different orientations. The generally higher von Mises stress values observed for soft inclusions are reflected in the corresponding inclusion factors ranging from 1.49 to 2.22.

In reality the amount, shape, size, and orientation of different kinds of inclusions depend very much on the steel making process [34]. However, it is well accepted that hard aluminium oxide inclusions are typically close to a spherical shape while manganese sulfide inclusions are often found as stringers of ellipsoidal form $[27,34]$. Therefore, the following part of the paper focuses on these realistic geometries for each inclusion type, instead of simply adopting the most critical cases from Table 2. 
Table 2: Maximum von Mises stresses for different inclusion types and inclusion factors with respect to the reference value $\sigma_{\mathrm{VM}}^{\max } / P_{H}=0.569$ in the absence of any inclusion.

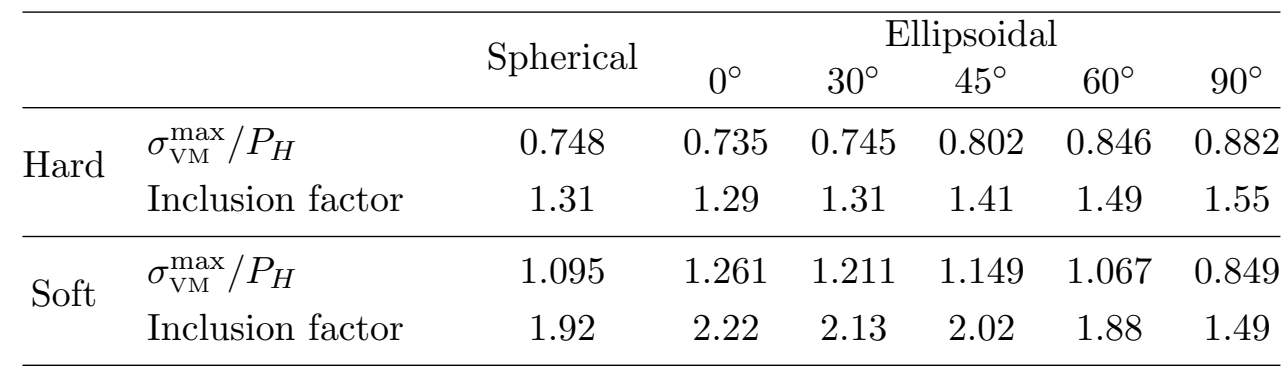

\subsection{Optimal residual stress profiles}

The procedure presented in section 2.2 is now applied to some selected cases of inclusion types and geometries in order to determine an optimal residual stress profile $\sigma_{\text {res }}^{*}(y)$ that will minimize the attained von Mises stresses. For this purpose, $\sigma_{\text {res }}$ is varied in the range of compressive stresses from $-800 \mathrm{MPa}\left(-0.35 P_{H}\right)$ to $0 \mathrm{MPa}$ in fixed increments of $5 \mathrm{MPa}\left(0.00217 P_{H}\right)$.

Figure 7 illustrates this optimization process through an example of a soft $0^{\circ}$ ellipsoidal inclusion at three arbitrary depths. The horizontal axis of the diagram corresponds to the probed range of compressive residual stresses $\sigma_{\text {res }}$, appearing in the outer min operator in Eq. (5), while the vertical axis shows the result of the two inner max operators. A positive effect of the superimposed residual stresses up to an optimal level around $-0.30 P_{H}$ is observed while there is a clear negative effect upon increasing compressive residual stresses beyond the detected optimum. The minima of the three plotted curves correspond to the sought optimal residual stresses $\sigma_{\text {res }}^{*}$ at the corresponding depths $y$. For better illustrating the above-mentioned nonmonotonic effect of compressive residual stresses, the horizontal axis is extended up to $-0.7 P_{H}$, beyond the limit of $-0.35 P_{H}$, which is otherwise used for extracting the results in the remaining part of the paper.

Figure 8 shows the original and optimized micro-scale von Mises stresses for hard spherical inclusions as well as the obtained optimal residual stress profile $\sigma_{\text {res }}^{*}(y)$. A significant reduction in the maximum von Mises stresses throughout the investigated depths can be observed.

A consequence of this result is that superposition of the obtained residual stress profiles will lead to a significant reduction of micro-scale plastic deformation during the running-in phase of the bearing operation. To the degree that micro-scale plasticity affects fatigue performance through nucle- 


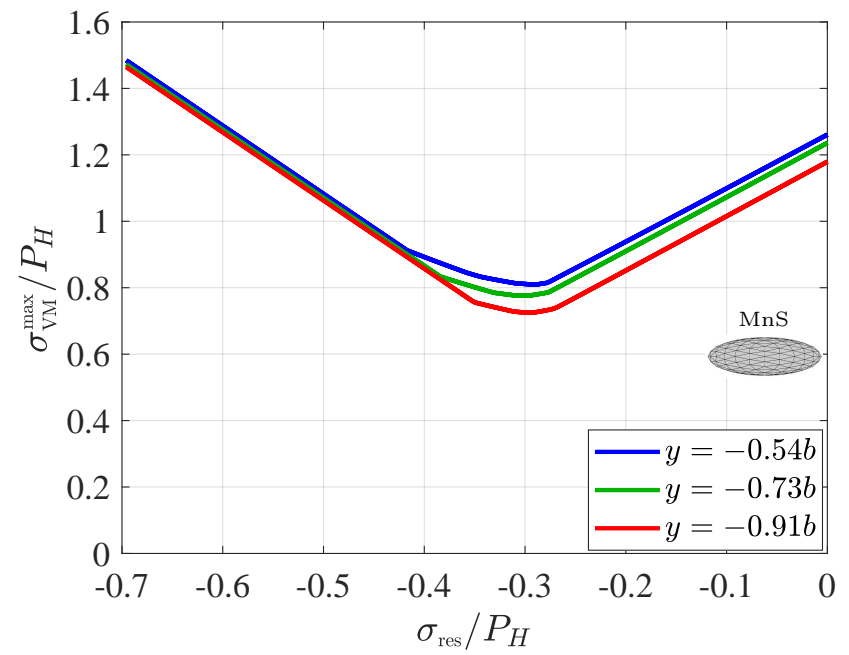

Figure 7: Effect of superimposed compressive residual stresses on the maximum von Mises stress around a soft $0^{\circ}$ ellipsoidal inclusion at arbitrary depths $y$.

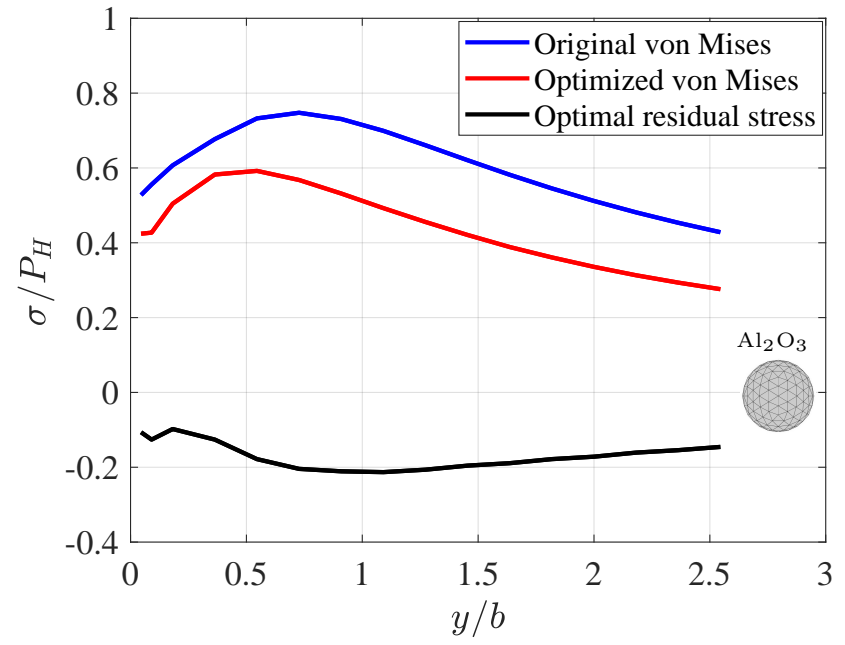

Figure 8: Optimal residual stress profiles along the depth direction and their effect on maximum von Mises stresses for a hard spherical inclusion.

ation of near inclusion cracks, the expected improvement will carry over to the component fatigue life.

It should be kept in mind that von Mises stress results presented so far are maximum values over the entire load cycle, as well as over space, covering a large number of discrete points around the inclusion. Moreover, the presented 
von Mises stress curves may attain their maximum at different depths $y$ from the raceway surface. Figure 9 illustrates the occurrence of the maximum von Mises stress for a hard spherical inclusion without and with residual stresses. In the former case, the maximum von Mises stress occurs approximately at a depth of $y=-0.73 b$ while in the latter case the corresponding maximum occurs around $y=-0.54 b$. Therefore, the stress histories shown on the left and right hand side of Figure 9, apart from the offsetting of $\sigma_{x x}$ and $\sigma_{z z}$ by $\sigma_{\text {res }}$, they also contain some additional differences due to the different considered depths.

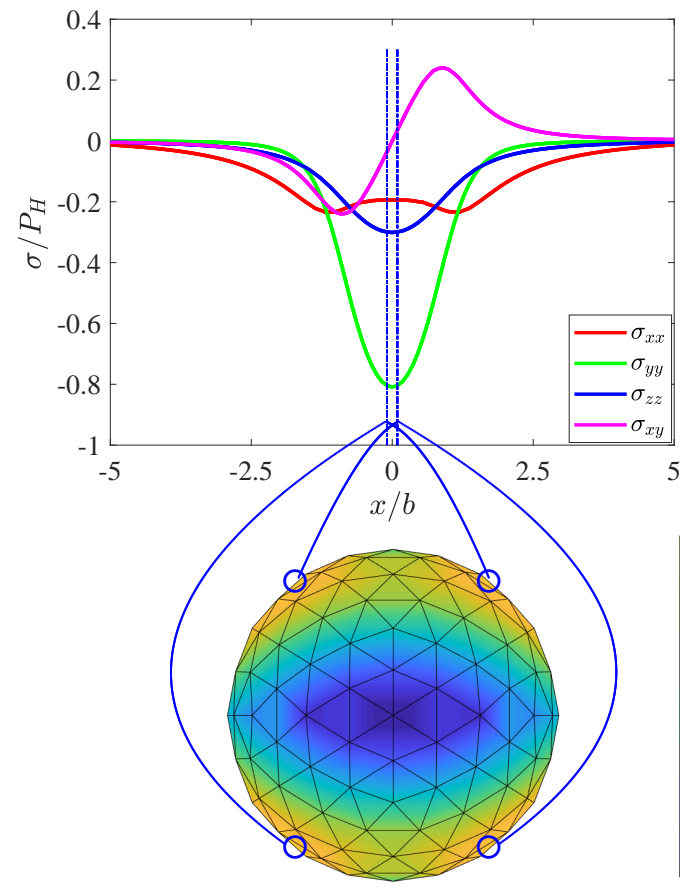

Without residual stress at $y=-0.73 b$

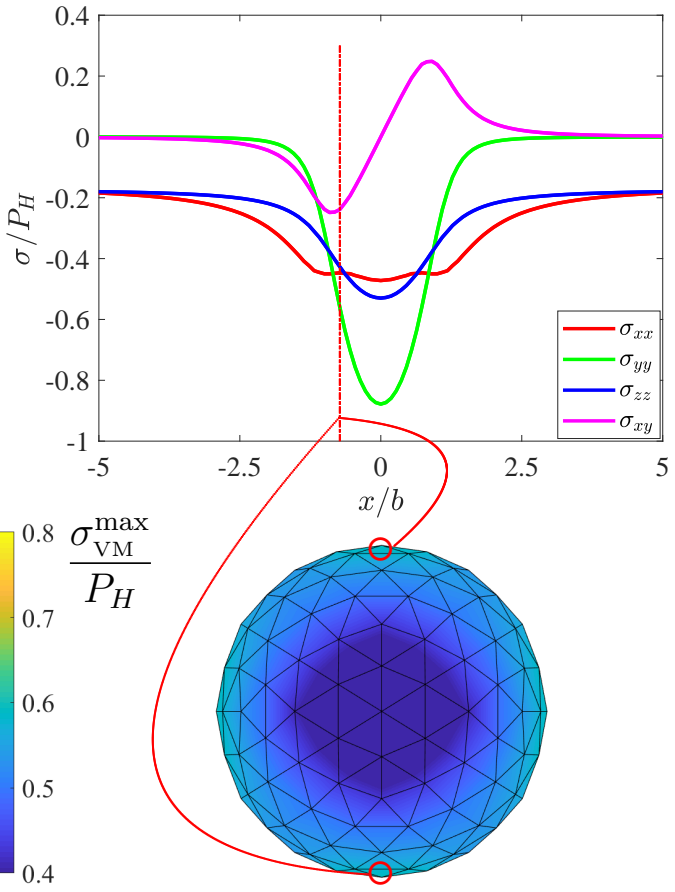

With optimal residual stress at $y=-0.54 b$

Figure 9: Spatial and temporal position of maximum micro-scale von Mises stresses for hard spherical inclusions, without and with residual stresses.

During a complete load cycle, the maximum von Mises stress is attained approximately when the $\sigma_{y y}$ component reaches its extremum for the case without any residual stresses. On the contrary, after adding the optimal residual stress, the corresponding maximum occurs approximately when the orthogonal shear stress component $\sigma_{x y}$ is at its peak. The plots at the bottom of the figure show the spatial distribution of the maximum von Mises stress 
close to the inclusion interface, attained over a complete load cycle. For the case without residual stresses, micro-scale plasticity is expected to initiate at the four locations marked on the left subfigure. On the other hand, with the added residual stress, the largest von Mises stresses occur at the poles of the inclusion although very similar equivalent stress levels are found along the whole meridian of the inclusion, in this case.

Corresponding results are now presented for soft inclusions of ellipsoidal shape. Figure 10 shows the optimal residual stresses obtained for soft ellipsoidal inclusions at $0^{\circ}$ and $90^{\circ}$ and its effect on the maximum micro-scale von Mises stresses. For soft inclusions elongated along the rolling direction, a considerable reduction is achieved over the complete range of depths. For the less critical case of soft $90^{\circ}$ ellipsoidal inclusions though, especially at very shallow depths, it is not possible to obtain any significant improvement.

Of course as mentioned earlier, inclusions of different forms and orientations occur in real materials stochastically. In that sense, optimizing for the most critical inclusion shape seems to be a logical option, but it is also interesting to cross-check the effect of the optimal residual stress profile obtained for one specific inclusion geometry on inclusions of different geometries. Adopting the optimal residual stress obtained for the most critical case of the soft $0^{\circ}$ ellipsoidal inclusion and cross-checking its effect on the behavior of a $90^{\circ}$ ellipsoidal inclusion leads to the results shown in Figure 11. The superimposed compressive residual stresses still reduce the maximum value of von Mises stresses at larger depths to some extent, but they have a significant negative effect at depths smaller than $0.8 b$. In this case, a compromise between the two optimal residual stress profiles reported in Figure 10 seems to be a more appropriate choice.

Figure 12 illustrates the occurrence of the maximum von Mises stress in space and time for the initially most critical soft ellipsoidal inclusion type with a $0^{\circ}$ orientation. In this case, both before and after adding compressive residual stresses, the maximum von Mises stress occurs approximately at the same depth around $y=-0.54 b$. It can also be deduced from the figure that the location of the maximum von Mises stress remains the same, i.e. on the equator of the inclusion in the middle along the ellipsoid's long axis. Nevertheless, this maximum occurs at different time instants during the load cycle as can be seen in the figure. It should be noted though that both before and after the superposition of residual stresses, von Mises stress values very close to the detected maximum are reached all along the equator of the inclusion. Hence, for this soft ellipsoidal inclusion, plastic deformation is 


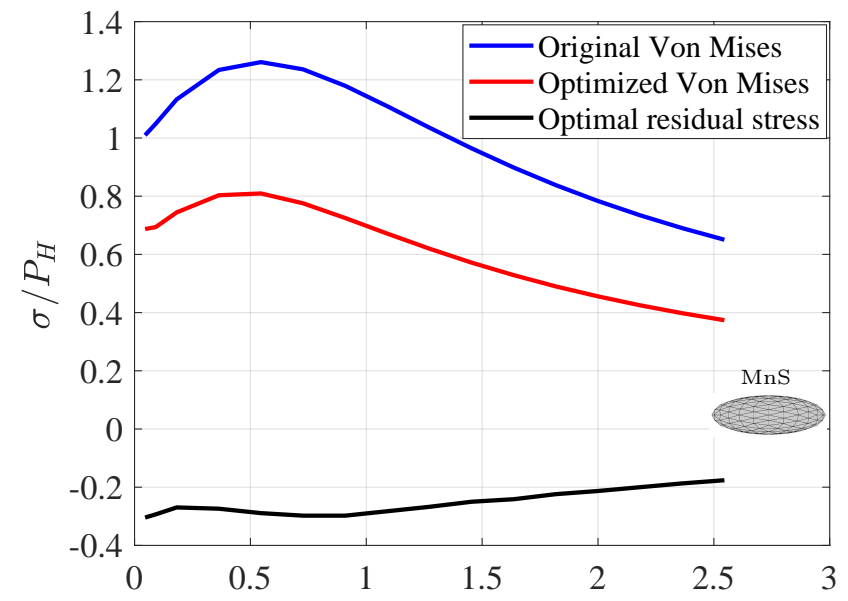

(a)

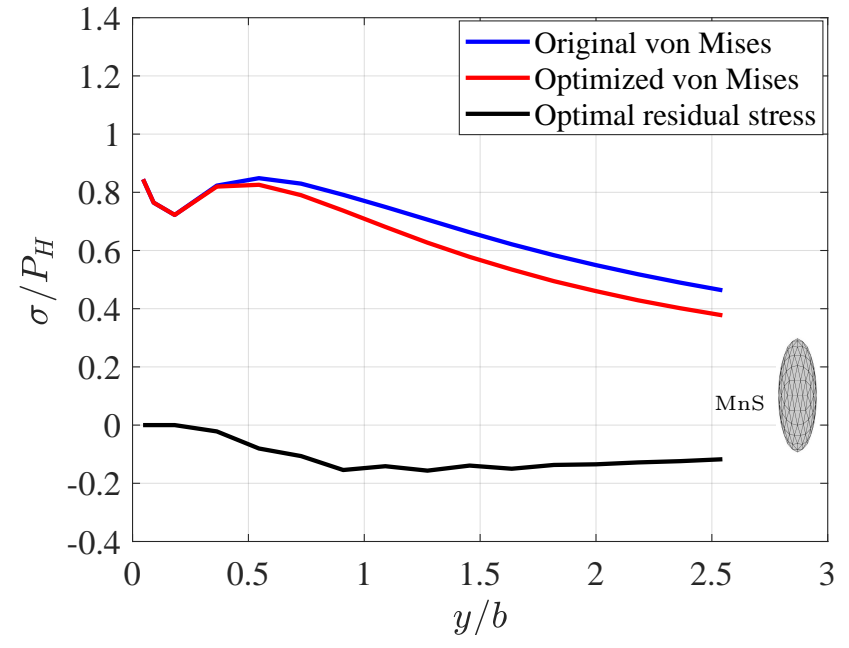

(b)

Figure 10: Optimal residual stress profiles along the depth direction and their effect on maximum von Mises stresss for soft ellipsoidal inclusions oriented at $0^{\circ}$ (a) and $90^{\circ}$ (b).

probably to occur within an annular region lying parallel to the raceway surface, i.e. in the $x z$ plane, irrespective of the presence of a macro-scale residual stress.

The presented results for both hard and soft inclusions have demonstrated that in most cases an optimal level of superimposed equibiaxial compressive residual stresses can be found in terms of avoiding initial micro-scale plasticity. As illustrated in Figure 7 for example, compressive stresses beyond the optimum point will lead to a reduced gain or even to a deterioration compared to the initial state. Hence, to the degree that initial micro-scale 


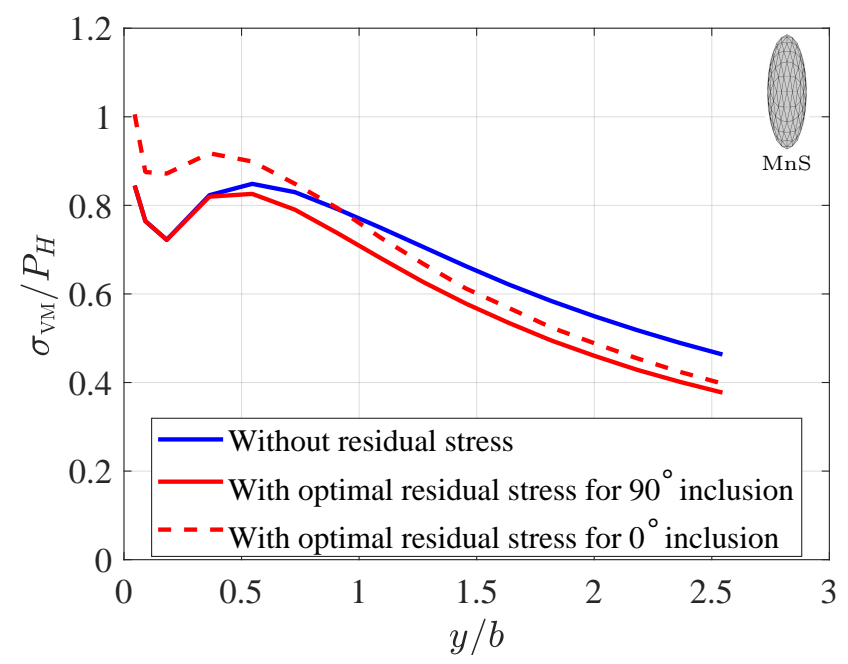

Figure 11: The effect of superimposed residual stresses optimized for soft $0^{\circ}$ ellipsoidal inclusions on the von Mises stresses for soft $90^{\circ}$ ellipsoidal inclusions.

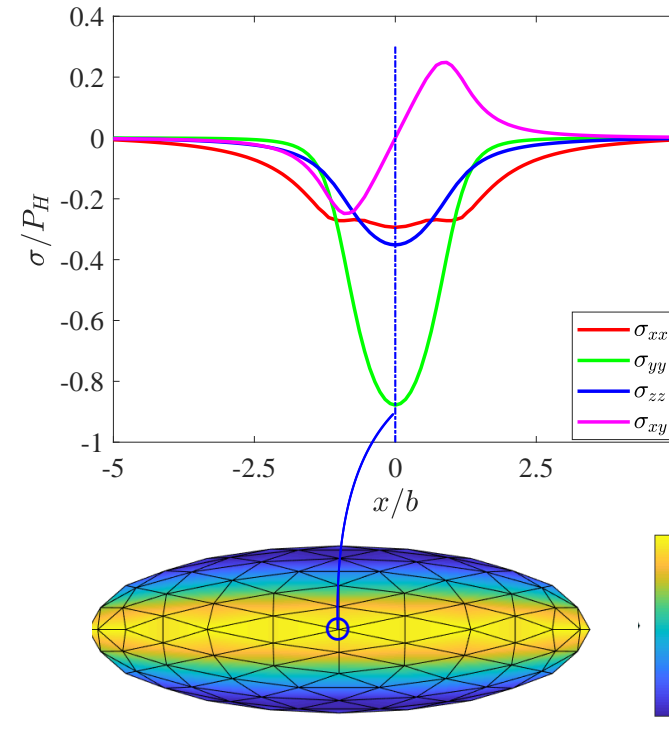

Without residual stresses

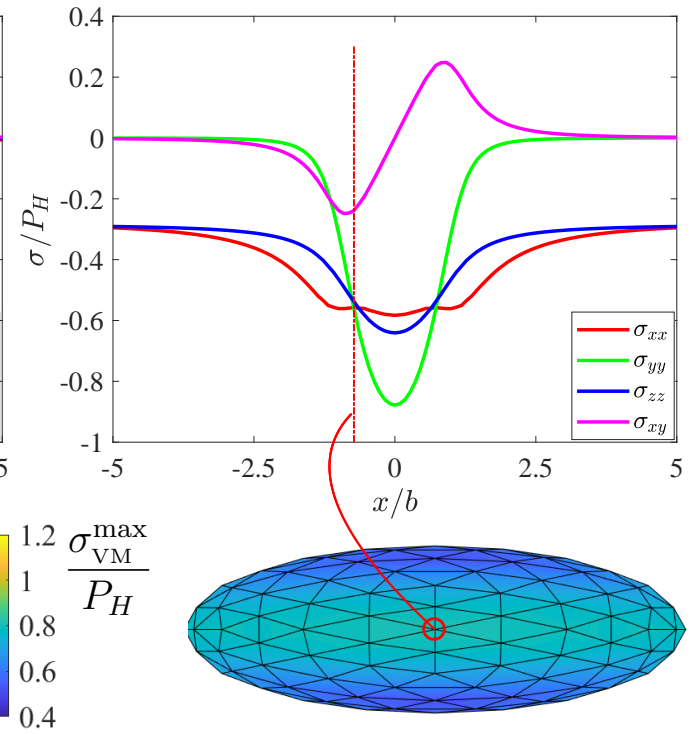

With optimal residual stress

Figure 12: Spatial and temporal position of maximum micro-scale von Mises stresses for soft $0^{\circ}$ ellipsoidal inclusions, without and with residual stresses.

plasticity can be linked to posterior fatigue behavior through nucleation of near inclusion micro-cracks, this outcome suggests that excessive compressive 
residual stresses can also compromise fatigue resistance.

Apart from the two types of inclusions investigated here, void imperfections may also be relevant for bearing steels. This case is omitted here as it leads to results similar to the case of soft inclusions. For hard inclusions, the results presented should be used with care since in reality as mentioned earlier, hard inclusions can be initially debonded from the matrix, leading to an entirely different behavior. In this case, a void model might be a better approximation of the defect site rather than a fully bonded inclusion.

As for the parabolic Hertzian load assumed in the present investigation, it can be seen as a practical compromise in the interest of simplicity as compared to a more realistic elastohydrodynamic pressure distribution. It is expected though that adoption of a more realistic load, possibly also with tangential traction components, will not affect the main conclusions of the presented investigations significantly.

Last, it is important to note the significant practical constraints regarding the through depth residual stress profiles that are feasible to implement. Even if tailored surface hardening processes are used for approximating a desired residual stress profile, overall equilibrium requires that compressive residual stresses close to the surface are in balance with tensile residual stresses at larger depths. In order to keep the introduced tensile residual stresses at larger depths as low as possible, it is hence appropriate to aim at the compressive residual stresses, proposed here, only up to a certain depth, e.g. $y=-b$, and allow a gradual decrease at larger depths. Accounting for the stochastic occurrence of inclusions of different shapes and orientations in the same type of component might also lead to necessary compromises. Based on the observation made in connection to Figure 11, it seems reasonable for instance to aim at an average between the two optimal residual stress profiles reported in Figure 10 for different inclusion orientations.

\section{Conclusions}

In this study, a semi-analytical method has been proposed for calculating favorable compressive residual stress profiles, aiming to suppress initial microscale plasticity around non-metallic inclusions in rolling contact fatigue. To the degree that micro-scale plasticity can be linked to the subsequent fatigue process, the superimposed residual stresses are expected to also have a positive effect on fatigue performance. 
According to the analysis of hard and soft inclusions of different geometries in the absence of macro-scale residual stresses, soft inclusions appear to be more critical with regard to causing micro-scale plasticity in their vicinity. Soft ellipsoidal inclusions elongated parallel to the rolling direction are the most critical among the investigated geometries. On the contrary, for hard inclusions, it is radially elongated ellipsoidal inclusions that lead to the highest von Mises stresses.

In most of the investigated cases, optimal compressive residual stresses could be obtained that lead to a remarkable reduction in the maximum von Mises stresses close to the considered inclusions. This is seen as a potential mechanism for improving the rolling contact fatigue performance of a component by avoiding crack nucleation due to initial plastic deformation. It should be noted though that this result also suggests that an excessive level of compressive residual stress can have a negative effect on fatigue performance, through the same mechanism.

An analysis of the distribution of von Mises stresses in space and its evolution through time has shown that macro-scale residual stresses can affect the occurrence of micro-scale plasticity both in space and time. In general, the subsurface depth of maximum micro-scale von Mises stresses changes after the addition of the optimal residual stress profiles. Moreover, in several cases, the site of the maximum von Mises stress around an inclusion is altered and its occurrence during a loading cycle is shifted.

Acknowledgements: This work is supported by the Innovation Fund Denmark grant with number 7046-00003B. The authors would also like to thank project collaborator Yuri Kadin from SKF for his valuable input.

\section{References}

[1] W. E. Littmann, The mechanism of contact fatigue, NASA Spec Report, SP-237 n.d.

[2] W. E. Littmann, R. L. Widner, Propagation of contact fatigue from surface and subsurface origins, American Society of Mechanical Engineers - Papers (1965).

[3] A. F. Bower, The influence of crack face friction and trapped fluid on surface initiated rolling-contact fatigue cracks, Journal of TribologyTransactions of the ASME 110 (1988) 704-711. 
[4] T. A. Harris, Rolling bearing analysis, Wiley, New York, 2001.

[5] J. M. Beswick, Fracture and fatigue crack propagation properties of hardened 52100 steel, Metallurgical Transactions A 20 (1989) 19611973.

[6] A. Gabelli, J. Lai, T. Lund, K. Rydn, I. Strandell, G. E. Morales-Espejel, The fatigue limit of bearing steels - Part II: Characterization for life rating standards, International Journal of Fatigue 38 (2012) 169-180.

[7] E. V. Zaretsky, J. V. Poplawski, S. M. Peters, Comparison of life theories for rolling-element bearings, Tribology Transactions 39 (1996) 501-503.

[8] ISO 281, Dynamic load ratings and rating life, Standard, International Organization for Standardization, (2007).

[9] N. G. Popinceanu, E. Diaconescu, S. Cretu, Critical stresses in rolling contact fatigue, Wear 71 (1981) 265-282.

[10] K. Dang-Van, Macro-micro approach in high-cycle multiaxial fatigue, Astm Special Technical Publication (1993) 120-130.

[11] H.-J. Böhmer, A new approach to determine the effect of nonmetallic inclusions on material behavior in rolling contact, Creative Use of Bearing Steels (1993) 211-221.

[12] J. Lai, T. Lund, K. Rydn, A. Gabelli, I. Strandell, The fatigue limit of bearing steels - Part I: A pragmatic approach to predict very high cycle fatigue strength, International Journal of Fatigue 38 (2012) 155-168.

[13] A. A. Walvekar, F. Sadeghi, Rolling contact fatigue of case carburized steels, International Journal of Fatigue 95 (2017) 264-281.

[14] A. P. Voskamp, E. J. Mittemeijer, The effect of the changing microstructure on the fatigue behaviour during cyclic rolling contact loading, Zeitschrift fuer Metallkunde 88 (1997) 310-320.

[15] G. Totten, M. Howes, T. Inoue, Handbook of Residual Stress and Deformation of Steel, ASM International, 2002. 
[16] A. Warhadpande, F. Sadeghi, R. D. Evans, M. N. Kotzalas, Influence of plasticity-induced residual stresses on rolling contact fatigue, Tribology Transactions 55 (2012) 422-437.

[17] R. K. Kepple, R. L. Mattson, Rolling element fatigue and macroresidual stress, Journal of Lubrication Technology 92 (1970) 76-81.

[18] Y. Shen, S. M. Moghadam, F. Sadeghi, K. Paulson, R. W. Trice, Effect of retained austenite - compressive residual stresses on rolling contact fatigue life of carburized AISI 8620 steel, International Journal of Fatigue 75 (2015) 135-144.

[19] J. Guan, L. Wang, Y. Mao, X. Shi, X. Ma, B. Hu, A continuum damage mechanics based approach to damage evolution of M50 bearing steel considering residual stress induced by shot peening, Tribology International 126 (2018) 218-228.

[20] S. Cretu, N. Popinceanu, The influence of residual-stresses induced by plastic-deformation on rolling-contact fatigue, Wear 105 (1985) 153-170.

[21] P. Lamagnere, R. Fougeres, G. Lormand, A. Vincent, D. Girodin, G. Dudragne, F. Vergne, A physically based model for endurance limit of bearing steels, Journal of Tribology 120 (1998) 421-426.

[22] C. R. Liu, S. Mittal, Optimal pre-stressing the surface of a component by superfinish hard turning for maximum fatigue life in rolling contact, Wear 219 (1998) 128-140.

[23] E. Ioannides, T. A. Harris, A new fatigue life model for rolling bearings, Journal of Tribology 107 (1985) 367-377.

[24] T. A. Harris, Prediction of ball fatigue life in a ball/V-ring test rig, Journal of Tribology-transactions of the ASME 119 (1997) 365-370.

[25] J. Courbon, G. Lormand, G. Dudragne, P. Daguier, A. Vincent, Influence of inclusion pairs, clusters and stringers on the lower bound of the endurance limit of bearing steels, Tribology International 36 (2003) 921-928.

[26] B. Allison, A. Pandkar, Critical factors for determining a first estimate of fatigue limit of bearing steels under rolling contact fatigue, International Journal of Fatigue 117 (2018) 396-406. 
[27] K. Hashimoto, T. Fujimatsu, N. Tsunekage, K. Hiraoka, K. Kida, E. C. Santos, Study of rolling contact fatigue of bearing steels in relation to various oxide inclusions, Materials and Design 32 (2011) 1605-1611.

[28] E. B. Pretorius, H. G. Oltmann, B. T. Schart, An overview of steel cleanliness from an industry perspective, Aistech - Iron and Steel Technology Conference Proceedings 1 (2013) 993-1026.

[29] A. Vincent, R. Fougeres, G. Lormand, G. Dudragne, D. Girodin, A physically based endurance limit model for through hardened and surface hardened bearing steels, ASTM Special Technical Publication (2002) 459-473.

[30] J. D. Eshelby, Elastic inclusions and inhomogeneities, Progress in Solid Mechanics 2 (1961) 89-140.

[31] T. Mura, Micromechanics of defects in solids, Martinus Nijhoff Publishers, 1982.

[32] C. Meng, W. Heltsley, D. D. Pollard, Evaluation of the Eshelby solution for the ellipsoidal inclusion and heterogeneity, Computers and Geosciences 40 (2012) 40-48.

[33] G. Popescu, A. Gabelli, G. M. Espejel, B. Wemekamp, Micro-plastic material model and residual fields in rolling contacts, Journal of ASTM International 3 (2006) 1-12.

[34] H. Fu, J. J. Rydel, A. M. Gola, F. Yu, K. Geng, C. Lau, H. Luo, P. E. J. Rivera-Daz-del-Castillo, The relationship between 100Cr6 steelmaking, inclusion microstructure and rolling contact fatigue performance, International Journal of Fatigue 129 (2019) 104899.

[35] F. Matsuno, S. Nishikida, H. Ikesaki, Mechanical properties of manganese sulphides in the temperature range between room temperature and $1000^{\circ} \mathrm{C}$, Transactions of the Iron and Steel Institute of Japan 25 (1985) 989-998.

[36] H. K. Bhadeshia, Steels for bearings, Progress in Materials Science 57 (2012) 268-435. 